\title{
Triangular fully packed loop configurations of excess 2
}

\author{
Sabine Beil
}

Universität Wien, Fakultät für Mathematik, Oskar-Morgenstern-Platz 1, 1090 Wien, Austria

\begin{abstract}
Triangular fully packed loop configurations (TFPLs) came up in the study of fully packed loop configurations on a square (FPLs) corresponding to link patterns with a large number of nested arches. To a TFPL is assigned a triple $(u, v ; w)$ of 01 -words encoding its boundary conditions. A necessary condition for the boundary $(u, v ; w)$ of a TFPL is $|\lambda(u)|+|\lambda(v)| \leq|\lambda(w)|$ where $\lambda(u)$ denotes the Young diagram associated with the 01-word $u$. Wieland gyration, on the other hand, was invented to show the rotational invariance of the numbers $A_{\pi}$ of FPLs corresponding to a given link pattern $\pi$. Later, Wieland drift was defined as the natural adaption of Wieland gyration to TFPLs. The main contribution of this article is a linear expression for the number of TFPLs with boundary $(u, v ; w)$ where $|\lambda(w)|-|\lambda(u)|-|\lambda(v)| \leq 2$ in terms of numbers of stable TFPLs that is TFPLs invariant under Wieland drift. These stable TFPLs have boundary $\left(u^{+}, v^{+} ; w\right)$ for words $u^{+}$and $v^{+}$such that $\lambda(u) \subseteq \lambda\left(u^{+}\right)$and $\lambda(v) \subseteq \lambda\left(v^{+}\right)$.
\end{abstract}

Résumé. Les configurations de boucles compactes triangulaires ("triangular fully packed loop configurations", ou TFPLs) sont apparues dans l'étude des configurations de boucles compactes dans un carré (FPLs) correspondant à des motifs de liaison avec un grand nombre d'arcs imbriqués. À chaque TPFL on associe un triplet $(u, v ; w)$ de mots sur $\{0,1\}$, qui encode ses conditions aux bords. Une condition nécessaire pour le bord $(u, v ; w)$ d'un TFPL est $|\lambda(u)|+|\lambda(v)| \leq|\lambda(w)|$, où $\lambda(u)$ désigne le diagramme de Young associé au mot $u$. D'un autre côté, la giration de Wieland a été inventée pour montrer l'invariance par rotation des nombres $A_{\pi}$ de FPLs correspondant à un motif de liaison donné $\pi$. Plus tard, la déviation de Wieland a été définie pour adapter de manière naturelle la giration de Wieland aux TFPLs. La contribution principale de cet article est une expression linéaire pour le nombre de TFPLs de bord $(u, v ; w)$, où $|\lambda(w)|-|\lambda(u)|-|\lambda(v)| \leq 2$, en fonction des nombres de TFPLs stables, i.e., les TFPLs invariants par déviation de Wieland. Ces TFPLs stables ont pour bord $\left(u^{+}, v^{+} ; w\right)$, avec $u^{+}$et $v^{+}$des mots tels que $\lambda(u) \subseteq \lambda\left(u^{+}\right)$et $\lambda(v) \subseteq \lambda\left(v^{+}\right)$.

Keywords: Triangular fully packed loop configurations, Wieland drift

\section{Introduction}

Triangular fully packed loop configurations (TFPLs) came up in the study of fully packed loop configurations on a square (FPLs) corresponding to a link pattern with a large number of nested arches in [2]. It soon turned out that TFPLs possess a number of nice properties, which made them worthy objects of study by themselves. To each TFPL are assigned three 01-words $u, v$ and $w$ that encode its boundary conditions. By the results of [5, 3] the boundary $(u, v ; w)$ of a TFPL has to fulfill that $|\lambda(w)|-|\lambda(u)|-|\lambda(v)|$ is a 
non-negative integer where $|\lambda(w)|$ denotes the number of cells of the Young diagram associated with $w$. The integer $|\lambda(w)|-|\lambda(u)|-|\lambda(v)|$ is said to be the excess of $u, v, w$.

In the case when the excess of $u, v, w$ vanishes, the number of TFPLs with boundary $(u, v ; w)$ is given by the Littlewood-Richardson coefficient $c_{\lambda(u), \lambda(v)}^{\lambda(w)}$, see [4] and [3]. Furthermore, in the case when the excess of $u, v, w$ equals 1 , a linear expression for the number of TFPLs with boundary $(u, v ; w)$ in terms of Littlewood-Richardson coefficients is given in [3]. For words $u, v, w$ of excess greater than 1 no enumeration results have been proved until now.

In this article, an enumeration result for TFPLs with boundary of excess 2 is presented which is a generalization of the above enumeration results for TFPLs with boundary of excess 0 and 1 . The key tool to derive this enumeration result is Wieland drift. In [1], Wieland drift was introduced as the natural definition of Wieland gyration for TFPLs. Wieland gyration itself was invented in [6] to prove the rotational invariance of the numbers $A_{\pi}$ of FPLs corresponding to a given link pattern $\pi$. While Wieland gyration for FPLs is an involution, Wieland drift is an eventually periodic map with period one, see [1]. In other words, the repeated application of Wieland drift to a TFPL ultimately leads to a TFPL that is invariant under Wieland drift; TFPLs that are invariant under Wieland drift are said to be stable whereas TFPL that are not invariant under Wieland drift are said to be instable. For instance, TFPLs with boundary $(u, v ; w)$ of excess 0 are stable.

The first contribution of this article is a detailed study of the effect of Wieland drift on instable TFPLs with boundary of excess at most 2 . The second contribution of this article is a linear expression for the numbers $t_{u, v}^{w}$ of TFPLs with boundary $(u, v ; w)$ of excess 2 in terms of the numbers $s_{u^{+}, v^{+}}^{w}$ of stable TFPLs with boundary $\left(u^{+}, v^{+} ; w\right)$ for $u^{+}$and $v^{+}$such that $\lambda(u) \subseteq \lambda\left(u^{+}\right)$and $\lambda(v) \subseteq \lambda\left(v^{+}\right)$.

Definition 1.1 Let $\lambda \subseteq \lambda^{+}$be two Young diagrams. The number of semi-standard Young tableaux of skew shape $\lambda^{+} / \lambda$ with entries in the $i$-th column, if counted from right, restricted to $1,2, \ldots, i$ is denoted by $g_{\lambda, \lambda^{+}}$.

Furthermore, given a Young diagram $\lambda$, its conjugate will be denoted by $\lambda^{\prime}$.

Theorem 1 Let $u, v, w$ be words of the same length such that $|\lambda(w)|-|\lambda(u)|-|\lambda(v)|=2$. Then

$$
t_{u, v}^{w}=\sum_{\substack{u^{+}: \lambda(u) \subseteq \lambda\left(u^{+}\right) \\ v^{+}: \lambda(v) \subseteq \lambda\left(v^{+}\right)}} g_{\lambda(u), \lambda\left(u^{+}\right)} g_{\lambda(v)^{\prime}, \lambda\left(v^{+}\right)^{\prime}} s_{u^{+}, v^{+}}^{w} .
$$

Since there does not exist a TFPL with boundary $(u, v ; w)$ unless $|\lambda(u)|+|\lambda(v)| \leq|\lambda(w)|$ the right hand side in 1.1 reduces to $s_{u, v}^{w}$ in the case when $|\lambda(u)|+|\lambda(v)|=|\lambda(w)|$. This is consistent with the fact that TFPLs with boundary $(u, v ; w)$ of excess 0 are stable; in other words, $s_{u, v}^{w}=t_{u, v}^{w}=c_{\lambda(u), \lambda(v)}^{\lambda(w)}$. Furthermore, if $|\lambda(w)|-|\lambda(u)|-|\lambda(v)|=1$ the linear expression obtained when subtracting $s_{u, v}^{w}$ from the right hand side of 1.1 coincides with the linear expression for instable TFPLs with boundary $(u, v ; w)$ in terms of Littlewood-Richardson coefficients proved in [3]. These observations encourage a detailed study of the effect of Wieland drift on TFPLs with boundary of excess greater than 2 in order to find linear expressions for their numbers in terms of stable TFPLs. 


\section{Preliminaries}

\subsection{Words and Young diagrams}

Throughout this article a word $\omega$ of length $N$ is a sequence $\omega=\omega_{1} \omega_{2} \cdots \omega_{N}$ where $\omega_{i} \in\{0,1\}$ for all $1 \leq i \leq N$. Given a word $\omega$ the number of occurrences of 0 (resp. 1) in $\omega$ is denoted by $|\omega|_{0}$ (resp. $\left.|\omega|_{1}\right)$. It is a well known fact that words $\omega$ satisfying $|\omega|_{1}=N_{1}$ and $|\omega|_{0}=N_{0}$ are in bijection with Young diagrams that fit into a rectangle consisting of $N_{1}$ columns and $N_{0}$ rows: given a word $\omega$ denote by $\lambda(\omega)$ the corresponding Young diagram. In Figure 1, an example of a word and its corresponding Young diagram is given. In the following, the number of cells of $\lambda(\omega)$ is denoted by $|\lambda(\omega)|$.
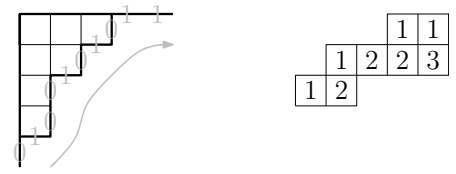

Fig. 1: The Young diagram $\lambda(0100101011)$ and a semi-standard Young tableau of skew shape $\lambda(11011100) / \lambda(01011011)$.

There are skew shaped Young diagrams which play an important role: a skew shape is said to be a horizontal strip (resp. a vertical strip) if each of its columns (resp. rows) contains at most one cell. In the following, if the skew shaped Young diagram $\lambda(\sigma) / \lambda(\omega)$ is a horizontal strip (resp. a vertical strip), it will be written $\omega \stackrel{\mathrm{h}}{\longrightarrow} \sigma$ (resp. $\omega \stackrel{\mathrm{v}}{\longrightarrow} \sigma$ ). Furthermore, semi-standard Young tableaux of skew shape $\lambda(\sigma) / \lambda(\omega)$ with entries $1,2, \ldots, m$ are in bijection with sequences of Young diagrams

$$
\lambda(\omega)=\lambda\left(\tau^{0}\right) \subseteq \lambda\left(\tau^{1}\right) \subseteq \cdots \subseteq \lambda\left(\tau^{m-1}\right) \subseteq \lambda\left(\tau^{m}\right)=\lambda(\sigma),
$$

such that $\tau^{i-1} \stackrel{\mathrm{h}}{\longrightarrow} \tau^{i}$ for each $1 \leq i \leq m$. For example, the semi-standard Young tableau of skew shape $\lambda(11011100) / \lambda(01011011)$ in Figure 1 corresponds to the sequence

$$
\lambda(01011011) \subseteq \lambda(10101110) \subseteq \lambda(11011010) \subseteq \lambda(11011100) .
$$

\subsection{Triangular fully packed loop configurations}

To give the definition of triangular fully packed loop configurations, the following graph is needed:

Definition 2.1 (The graph $G^{N}$ ) Let $N$ be a positive integer. The graph $G^{N}$ is defined as the induced subgraph of the square grid made up of $N$ consecutive centered rows of $3,5, \ldots, 2 N+1$ vertices from top to bottom together with $2 N+1$ vertical external edges incident to the $2 N+1$ bottom vertices.

From now on, the vertices of $G^{N}$ are partitioned into odd and even vertices in a chessboard manner where by convention the leftmost vertex of the top row of $G^{N}$ is odd. In the figures, odd vertices are represented by circles and even vertices by squares. There are vertices of $G^{N}$ that play a special role: let $\mathcal{L}^{N}=\left\{L_{1}, L_{2}, \ldots, L_{N}\right\}$ (resp. $\mathcal{R}^{N}=\left\{R_{1}, R_{2}, \ldots, R_{N}\right\}$ ) be the set made up of the vertices which are leftmost (resp. rightmost) in each of the $N$ rows of $G^{N}$ and let $\mathcal{B}^{N}=\left\{B_{1}, B_{2}, \ldots, B_{N}\right\}$ be the set made up of the even vertices of the bottom row of $G^{N}$. The vertices are numbered from left to right. Furthermore, the $N(N+3)$ unit squares of $G^{N}$, including external unit squares that have three 
surrounding edges only, are said to be the cells of $G^{N}$. They are partitioned into odd and even cells in a chessboard manner where by convention the top left cell of $G^{N}$ is odd.

Definition 2.2 (Triangular fully packed loop configuration) Let $N$ be a positive integer. A triangular fully packed loop configuration (TFPL) of size $N$ is a subgraph $f$ of $G^{N}$ such that:

1. Precisely those external edges that are incident to a vertex in $\mathcal{B}^{N}$ belong to $f$.

2. The $2 N$ vertices in $\mathcal{L}^{N} \cup \mathcal{R}^{N}$ have degree 0 or 1 .

3. All other vertices of $G^{N}$ have degree 2 .

4. A path in $f$ neither connects two vertices of $\mathcal{L}^{N}$ nor two vertices of $\mathcal{R}^{N}$.
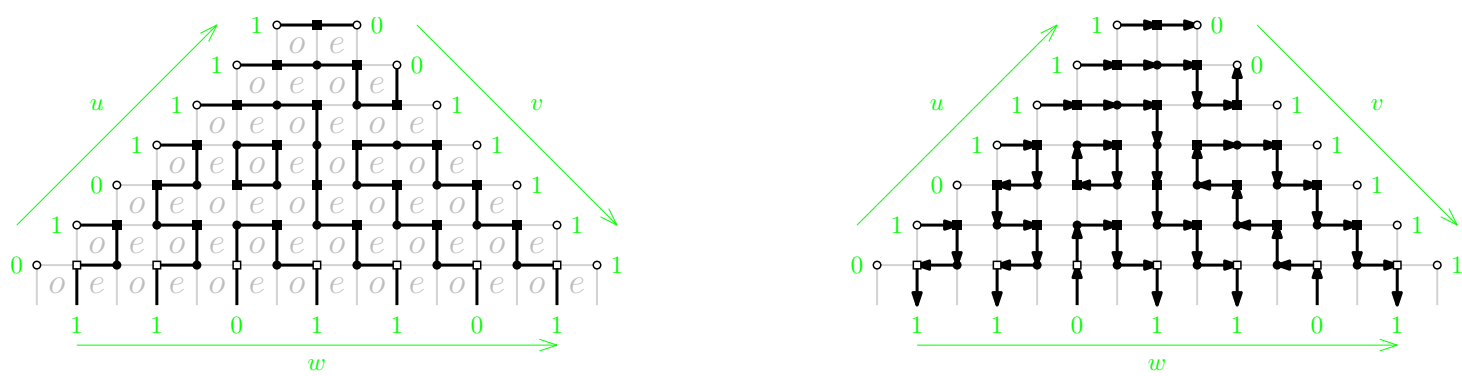

Fig. 2: Left: a TFPL of size 7 with boundary $(0101111,0011111 ; 1101101)$; right: the same TFPL together with its canonical orientation.

An example of a TFPL is given in Figure 2. A cell of $f$ is a cell of $G^{N}$ together with those of its surrounding edges that belong to $f$. To each TFPL of size $N$ a triple of words of length $N$ is assigned as follows:

Definition 2.3 Let $f$ be a TFPL of size $N$. A triple $(u, v ; w)$ of words of length $N$ is said to be the boundary of $f$ if for each $i=1,2, \ldots, N$ the following hold:

1. If the vertex $L_{i} \in \mathcal{L}^{N}$ has degree 1 , then $u_{i}=1$, otherwise, $u_{i}=0$.

2. If the vertex $R_{i} \in \mathcal{R}^{N}$ has degree 1 , then $v_{i}=0$, otherwise, $v_{i}=1$.

3. If the vertex $B_{i} \in \mathcal{B}^{N}$ is connected by a path either with a vertex in $\mathcal{L}^{N}$ or with a vertex $B_{h}$ where $h<i$ in $f$, then $w_{i}=1$, otherwise, $w_{i}=0$.

The set of all TFPLs with boundary $(u, v ; w)$ is denoted by $T_{u, v}^{w}$ and its cardinality by $t_{u, v}^{w}$.

A triple $(u, v ; w)$ that is the boundary of a TFPL has to fulfill $|u|_{0}=|v|_{0}=|w|_{0}$ and $|\lambda(u)|+|\lambda(v)| \leq$ $|\lambda(w)|$. These conditions came up in [2, 5]. The last condition gives rise to the following definition:

Definition 2.4 Let $u, v, w$ be words of length $N$. The excess of $u, v, w$ is defined as

$$
\operatorname{exc}(u, v ; w)=|\lambda(w)|-|\lambda(u)|-|\lambda(v)| .
$$

If $\operatorname{exc}(u, v ; w)=k$ a TFPL with boundary $(u, v ; w)$ is said to be of excess $k$. 
For the proofs of the results presented in this article it is crucial to consider TFPLs together with a canonical orientation of its edges; the canonical orientation of a TFPL is defined as the orientation of its edges that satisfies the following: each edge attached to a vertex in $\mathcal{L}^{N}$ is outgoing; each edge attached to a vertex in $\mathcal{R}^{N}$ is incoming; each other vertex is incident to an incoming and an outgoing edge; a path that connects two vertices $B_{i}$ and $B_{j}$ in $\mathcal{B}^{N}$ is oriented from $B_{i}$ to $B_{j}$ if $i<j$; each closed path is oriented clockwise. An example of a TFPL together with its canonical orientation is given in Figure 2 Considering a TFPL together with its canonical orientation allows the following combinatorial interpretation of the excess of the TFPL in terms of numbers of occurrences of certain local configurations:

Proposition 2.5 Let $f$ be a TFPL with boundary $(u, v ; w)$. When $f$ is regarded together with its canonical orientation the following holds:

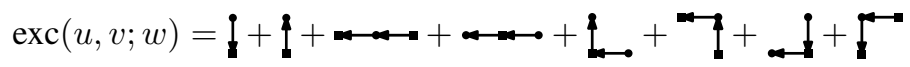

where by $\downarrow$, etc. the numbers of occurrences of the local configurations $\downarrow, \downarrow$, etc. are denoted.

Proposition 2.5 is shown in [3] for a more general case that is for TFPLs together with an orientation of their edges that satisfies that each edge attached to a vertex in $\mathcal{L}^{N}$ is outgoing, each edge attached to a vertex in $\mathcal{R}^{N}$ is incoming and each other vertex is incident to an incoming and an outgoing edge. Proposition 2.5 will be essential for the determination of the effect of Wieland drift on TFPLs of excess at most 2 presented in the next section.

\subsection{Wieland drift}

The starting point of this subsection is the definition of Wieland gyration for fully packed loop configurations (FPLs) as introduced in [6]. Wieland gyration $\mathrm{W}$ is composed of local operations on all active cells of an FPL; the active cells of an FPL can be chosen to be either all its odd cells or all its even cells. Given an active cell $c$ of an FPL two cases have to be distinguished, namely whether $c$ contains precisely two edges of the FPL on opposite sides or not. If this is the case W leaves $c$ invariant. Otherwise, the effect of $\mathrm{W}$ on $c$ is that edges and non-edges of the FPL are exchanged.

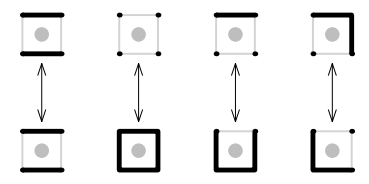

Fig. 3: Up to rotation, the action of $\mathrm{W}$ on an active cell of an FPL.

Wieland drift will be composed of local operations on all active cells of a TFPL. Choosing all odd cells as active cells will lead to what will be defined as left-Wieland drift, whereas choosing all even cells as active cells will lead to what will be defined as right-Wieland drift. In the figures the active cells of a TFPL will be indicated by gray circles.

Definition 2.6 (Left-Wieland drift) Let $f$ be a TFPL with left boundary word $u$ and let $u^{-}$be a word satisfying $u^{-} \stackrel{\mathrm{h}}{\longrightarrow} u$. The image of $f$ under left-Wieland drift with respect to $u^{-}$is determined as follows: 
1. Insert a vertex $L_{i}^{\prime}$ to the left of $L_{i}$ for $1 \leq i \leq N$. Then run through the occurrences of ones in $u^{-}$: Let $\left\{i_{1}<i_{2}<\ldots<i_{N_{1}}\right\}=\left\{i \mid u_{i}^{-}=1\right\}$.

(a) If $u_{i_{j}}$ is the $j$-th one in $u$, add a horizontal edge between $L_{i_{j}}^{\prime}$ and $L_{i_{j}}$.

(b) If $u_{i_{j}-1}$ is the $j$-th one in $u$, add a vertical edge between $L_{i_{j}}^{\prime}$ and $L_{i_{j}-1}$.

2. Apply Wieland gyration to each odd cell of $f$.

3. Delete all vertices in $\mathcal{R}^{N}$ and their incident edges.

After shifting the whole construction one unit to the right, one obtains the desired image $\mathrm{WL}_{u^{-}}(f)$; in the case $u^{-}=u$ simply write $\mathrm{WL}(f)$ and say the image of $f$ under left-Wieland drift.

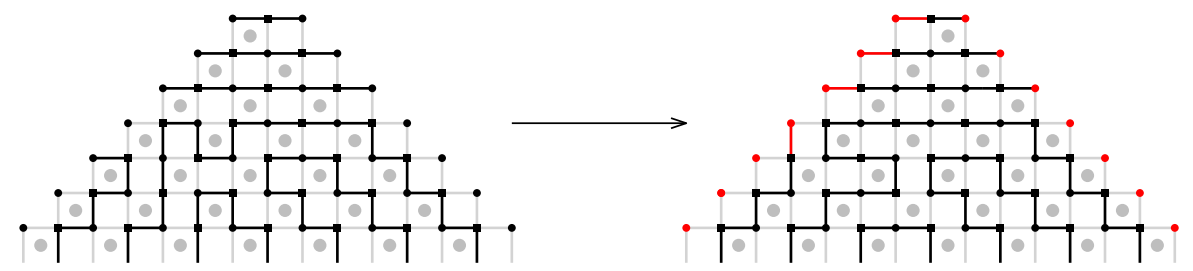

Fig. 4: A TFPL and its image under left-Wieland drift with respect to 0001111.

In Figure 4 an example for left-Wieland drift is given. The image of a TFPL with boundary $(u, v ; w)$ under left-Wieland drift with respect to $u^{-}$is again a TFPL and has boundary $\left(u^{-}, v^{+} ; w\right)$, where $v^{+}$is a word satisfying $v \stackrel{\mathrm{v}}{\longrightarrow} v^{+}$, see [1, Proposition 2.2].

Right-Wieland drift on the other hand depends on a word $v^{-}$satisfying $v^{-} \stackrel{\mathrm{v}}{\longrightarrow} v$ that encodes what happens along the right boundary of a TFPL with right boundary $v$ and is denoted by $\mathrm{WR}_{v^{-}}$respectively WR if $v^{-}=v$. It is defined in an obvious way as the symmetric version of left-Wieland drift. The image of a TFPL with boundary $(u, v ; w)$ under right-Wieland drift with respect to $v^{-}$is a TFPL with boundary $\left(u^{+}, v^{-} ; w\right)$ where $u^{+}$is a word satisfying $u \stackrel{\mathrm{h}}{\longrightarrow} u^{+}$.

Proposition 2.7 ([1]) Let $f$ be a TFPL with boundary $(u, v ; w), u^{-}$be a word such that $u^{-} \stackrel{\mathrm{h}}{\longrightarrow} u$ and $v^{-}$be a word such that $v^{-} \stackrel{\mathrm{v}}{\longrightarrow} v$. Then $\mathrm{WR}_{v}\left(\mathrm{WL}_{u^{-}}(f)\right)=\mathrm{WL}_{u}\left(\mathrm{WR}_{v^{-}}(f)\right)=f$.

By Proposition 2.7. a TFPL is invariant under left-Wieland drift if and only if it is invariant under right-Wieland drift. Hence, a TFPL is said to be stable if it is invariant under left-Wieland drift, whereas otherwise it is said to be instable. The set of stable TFPLs with boundary $(u, v ; w)$ is denoted by $S_{u, v}^{w}$ and its cardinality by $s_{u, v}^{w}$. In [1] the following characterization of stable TFPLs is shown:

Proposition 2.8 A TFPL is stable if and only if it contains no edge of the form !; such an edge is said to be $a$ drifter.

The number of drifters occuring in an instable TFPL is bounded by the excess of the TFPL:

Proposition 2.9 By Proposition 2.5 an instable TFPL of excess $k$ exhibits at most $k$ drifters. 
While the usual Wieland gyration of FPLs is an involution, Wieland drift is not. By a finiteness argument, the sequences $\left(\mathrm{WL}^{m}(f)\right)_{m>0}$ and $\left(\mathrm{WR}^{m}(f)\right)_{m>0}$ are eventually periodic for each TFPL $f$. In fact, the length of their periods is always 1 which is proved in [1]. Therefore, given a TFPL $f$ there exist non-negative integers $L(f)$ and $R(f)$ such that $\mathrm{WL}^{\ell}(f)$ is instable for each $0 \leq \ell \leq L(f)$ whereas $\mathrm{WL}^{L(f)+1}(f)$ is stable and $\mathrm{WR}^{r}(f)$ is instable for each $0 \leq r \leq R(f)$ whereas $\mathrm{WR}^{R(f)+1}(f)$ is stable. From now on, $\mathrm{WL}^{L(f)+1}(f)$ is denoted by $\operatorname{Left}(f)$ and $\mathrm{WR}^{R(f)+1}(f)$ by $\operatorname{Right}(f)$.

\section{Wieland drift for TFPLs of excess at most 2}

In this section the effect of Wieland drift on instable TFPLs of excess at most 2 is studied. The thereby obtained results generalize already existing results for TFPLs of excess 1 in [3] and [1]; the first such result will be a description of the effect of Wieland drift on instable TFPLs of excess at most 2 as a composition of moves. In Figure 5 the moves which form the basis for that description are depicted. The first three moves in the figure already appeared in the study of TFPLs of excess 1 in [3]; there they were invented by the authors in order to obtain enumeration results for TFPLs of excess 1. Later the author together with the authors of [3] realized that these moves are closely related to Wieland drift; by the application of Wieland drift to an instable TFPL of excess 1 one of the moves introduced in [3] is performed while the rest of the TFPL is preserved, see [1].
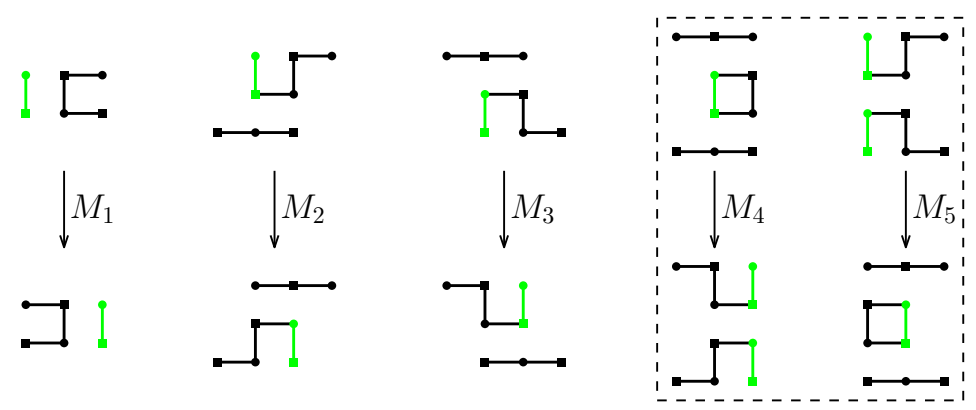

Fig. 5: The moves performed by left-Wieland drift to instable TFPLs of excess at most 2 with the moves that can only be performed to instable TFPLs of excess 2 indicated.

Proposition 3.1 Let $f$ be an instabe TFPL with boundary $(u, v ; w)$ such that $\operatorname{exc}(u, v ; w) \leq 2$ and $u^{-}$ be a word such that $u^{-} \stackrel{\mathrm{h}}{\rightarrow} u$ and $\operatorname{exc}\left(u^{-}, v ; w\right) \leq 2$. Then the effect of left-Wieland drift with respect to $u^{-}$on $f$ is determined as follows:

1. For each $i=1,2, \ldots, N$ such that $R_{i} \in \mathcal{R}^{N}$ is incident to a drifter delete the drifter and add a horizontal edge incident to $R_{i+1} \in \mathcal{R}^{N}$.

2. Either perform the move $M_{5}$ or apply a uniquely determined move in $\left\{M_{1}, M_{2}, M_{3}, M_{4}\right\}$ to each drifter following the subsequent order if there are two drifters: if the two drifters do not have the same $x$-coordinate the drifter with the larger x-coordinate is moved first; otherwise, the upper drifter is moved first if it is not contained in a cell of the form $\square$ whereas the lower drifter is moved first if the upper one is contained in a cell of the form $\square$. 
3. For each $j$ such that $u_{i_{j}}^{-}$is the $j$-th one in $u^{-}$and $u_{i_{j}-1}$ is the $j$-th one in $u$ delete the horizontal edge incident to $L_{i_{j}-1}$ and add a drifter incident to $L_{i_{j}}$.

To prove Proposition 3.1 one has to check the effect of left-Wieland drift on a TFPL of excess at most 2 cell by cell; thereby it suffices to check the cells around a drifter due to Lemma 20 in [1]. In order to determine which cells may occur near a drifter in a TFPL of excess at most 2 one needs to regard the TFPL together with its canonical orientation and to use the combinatorial interpretation of the excess given in Proposition 2.5. A description of the effect of right-Wieland drift on instable TFPLs of excess at most 2 as a composition of moves can be derived from Proposition 3.1 and Proposition 2.7. The moves on which this description is based are the inverse moves $M_{1}^{-1}, M_{2}^{-1}, M_{3}^{-1}, M_{4}^{-1}, M_{5}^{-1}$ of the moves in Figure 5

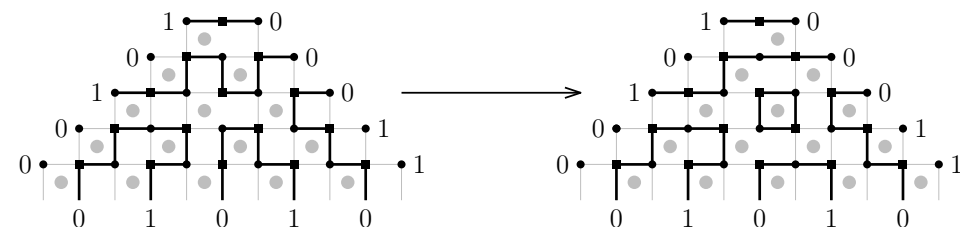

Fig. 6: A TFPL of excess 2 and its image under left-Wieland drift.

The next result will be a formula for the sum of the number of iterations of WL that are needed to move a drifter $\mathfrak{d}$ in an instable TFPL $f$ to the right boundary and the number of iterations of WR that are needed to move $\mathfrak{d}$ to the left boundary. This formula will be essential for the proof of the main result of this article which is stated in Theorem 1 . When looking at the moves that describe the effect of Wieland drift on instable TFPLs of excess at most 2 one immediately sees that in the preimage of the move $M_{4}$ there is one drifter whereas in its image there are two and that in the preimage of the move $M_{5}$ there are two drifters whereas in its image there is one. This is why in order to pursue a drifter under an iterated application of Wieland drift one has to decide which drifter to pursue after applying the move $M_{5}$ resp. $M_{4}^{-1}$. Hence, fix a drifter in the image of the move $M_{5}^{-1}$ that is identified with the drifter in the preimage of the move $M_{5}^{-1}$ and fix a drifter in the image of the move $M_{4}$ that is identified with the drifter in the preimage of the move $M_{4}$.

Definition 3.2 $\left(\operatorname{Path}(f)_{\mathfrak{d}}, \operatorname{Left}(f)_{\mathfrak{d}}, \operatorname{Right}(f)_{\mathfrak{d}}\right.$, HeightL $\left.(f)_{\mathfrak{d}}, \operatorname{HeightR}(f)_{\mathfrak{d}}\right)$ Let $f$ be an instable TFPL of excess at most 2 and $\mathfrak{d}$ a drifter in $f$.

- The path of $\mathfrak{d}$, denoted by $\operatorname{Path}(f)_{\mathfrak{d}}$, is the sequence of all instable TFPLs that contain $\mathfrak{d}$ and can be reached by a repeated application of left- resp. right-Wieland drift to $f$.

- The unique TFPL in $\operatorname{Path}(f)_{\mathfrak{d}}$ with $\mathfrak{d}$ incident to a vertex in $\mathcal{L}^{N}$ is denoted by $\operatorname{Right}(f)_{\mathfrak{d}}$; Left $(f)_{\mathfrak{d}}$ is defined analogously.

- The unique positive integer $h$ with $\mathfrak{d}$ incident to $L_{h+1}$ in $\operatorname{Right}(f)_{\mathfrak{d}}$ is denoted by $\operatorname{HeigthR}(f)_{\mathfrak{d}}$ and the unique positive integer $h^{\prime}$ with $\mathfrak{d}$ incident to $R_{N-h^{\prime}}$ in $\operatorname{Left}(f)_{\mathfrak{d}}$ is denoted by $\operatorname{HeightL}(f)_{\mathfrak{D}}$. 
By definition, there exist non-negative integers $L(f)_{\mathfrak{d}}$ and $R(f)_{\mathfrak{d}}$ such that $\operatorname{Right}(f)_{\mathfrak{d}}=\mathrm{WR}^{R(f)_{\mathfrak{o}}}(f)$ and $\operatorname{Left}(f)_{\mathfrak{o}}=\mathrm{WL}^{L(f)_{\mathfrak{o}}}(f)$. Therefore, $\left|\operatorname{Path}(f)_{\mathfrak{o}}\right|=L(f)_{\mathfrak{d}}+R(f)_{\mathfrak{o}}+1$. In the following, set $\# M_{i}(f)_{\mathfrak{d}}=\#\left\{1 \leq \ell \leq L(f)_{\mathfrak{d}} \mid\right.$ the move $M_{i}$ is applied to $\mathfrak{d}$ by the application of WL to $\left.\mathrm{WL}^{\ell-1}(f)\right\}$ $+\#\left\{1 \leq r \leq R(f)_{\mathfrak{d}} \mid\right.$ the move $M_{i}^{-1}$ is applied to $\mathfrak{d}$ by the application of WR to $\left.\mathrm{WR}^{r-1}(f)\right\}$

Thus, $\left|\operatorname{Path}(f)_{\mathfrak{d}}\right|=\# M_{1}(f)_{\mathfrak{o}}+\# M_{2}(f)_{\mathfrak{d}}+\# M_{3}(f)_{\mathfrak{d}}+\# M_{4}(f)_{\mathfrak{d}}+\# M_{5}(f)_{\mathfrak{d}}+1$.

Definition $3.3\left(R_{i}\left(u^{R(f)_{\mathfrak{}}}\right), L_{i}\left(v^{L(f)_{\mathfrak{o}}}\right)\right)$ Let $u, v, w$ be words such that exc $(u, v ; w) \leq 2, f$ an instable TFPL with boundary $(u, v ; w)$ and $\mathfrak{d}$ a drifter in $f$. When $u^{R(f)_{0}}$ denotes the left boundary word of $\mathrm{WR}^{R(f)_{0}}(f)$ and $v^{L(f)_{\mathrm{o}}}$ denotes the right boundary word of $\mathrm{WL}^{L(f)_{0}}(f)$, then define $R_{i}\left(u^{R(f)_{0}}\right)$ as the number of occurrences of $i$ among the last $\left(N-1-\operatorname{HeightR}(f)_{\mathfrak{o}}\right)$ letters of $u^{R(f)_{\mathfrak{o}}}$ and $L_{i}\left(v^{L(f)_{\mathfrak{o}}}\right)$ as the number of occurrences of $i$ among the first $\left(N-1-\operatorname{Height} L(f)_{\mathfrak{d}}\right)$ letters of $v^{L(f)_{\mathrm{o}}}$ for $i=0,1$.
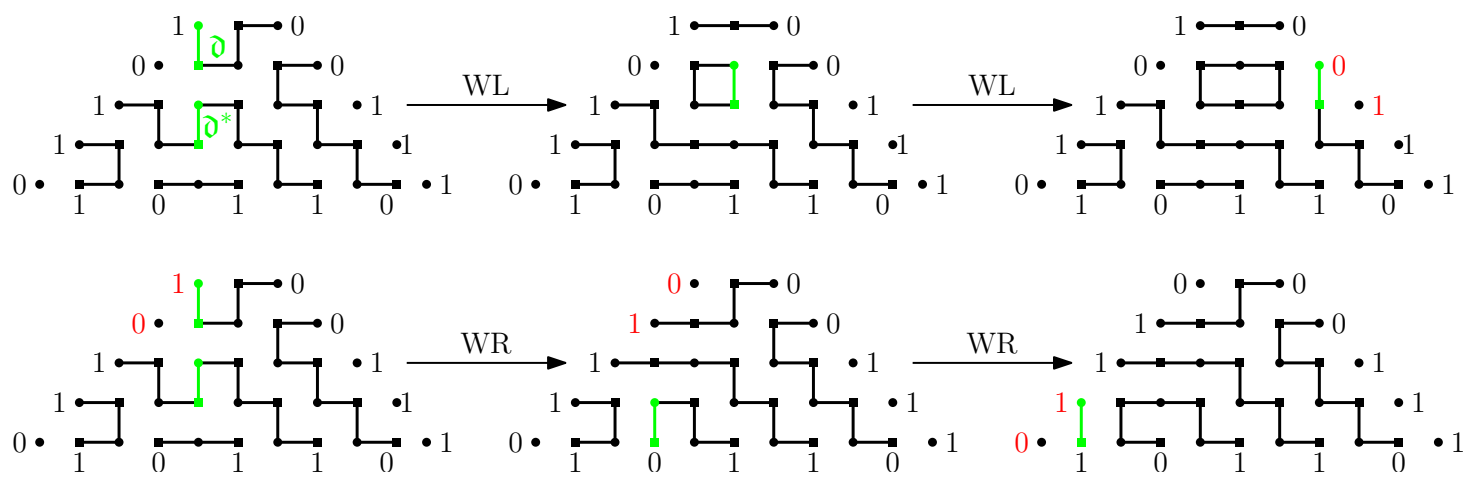

Fig. 7: A TFPL of excess 2 and the instable TFPLs obtained from it by a repeated applications of left- resp. rightWieland drift. Here, $L(f)_{\mathfrak{o}}=L(f)_{\mathfrak{d}^{*}}=2, R(f)_{\mathfrak{o}}=0$ and $R(f)_{\mathfrak{o}^{*}}=2$.

For the example depicted in Figure 7 , it holds $L_{0}\left(v^{L(f)_{\triangleright}}\right)=L_{0}\left(v^{L(f)_{\mathrm{\vartheta}^{*}}}\right)=1, R_{1}\left(u^{R(f)_{\triangleright}}\right)=0$ and $R_{1}\left(u^{R(f)_{\mathfrak{o}^{*}}}\right)=2$.

Proposition 3.4 Let $f$ be an instable TFPL with boundary $(u, v ; w)$, where exc $(u, v ; w) \leq 2$, $\mathfrak{d}$ a drifter in $f$ and the notations as above. Then

$$
\# M_{1}(f)_{\mathfrak{o}}+\# M_{2}(f)_{\mathfrak{o}}+\# M_{3}(f)_{\mathfrak{o}}+\# M_{4}(f)_{\mathfrak{o}}+\# M_{5}(f)_{\mathfrak{o}}=R_{1}\left(u^{R(f)_{\mathfrak{o}}}\right)+L_{0}\left(v^{L(f)_{\mathfrak{o}}}\right)+1 .
$$

In particular, $L(f)_{\mathfrak{o}}+R(f)_{\mathfrak{o}}=R_{1}\left(u^{R(f)_{\mathfrak{o}}}\right)+L_{0}\left(v^{L(f)_{\mathfrak{o}}}\right)+1$.

In [3], it is shown that oriented TFPLs are in bijection with certain non-intersecting lattice paths, so-called blue-red path tangles. The fundamental idea for the proof of Proposition 3.4 is to regard the blue-red path tangles corresponding to TFPLs of excess at most 2 together with their canonical orientation. Then analogous arguments as in the proofs of Proposition 6.11 and Proposition 6.12 in [3] can be used to prove Proposition 3.4 . 


\section{Proof of Theorem 1}

In this section, a bijective proof of Theorem 1 is given. By Proposition 3.4 precisely one of the inequalities

$$
L(f)_{\mathfrak{d}} \leq L_{0}\left(v^{L(f)_{\mathfrak{o}}}\right) \text { and } R(f)_{\mathfrak{d}} \leq R_{1}\left(u^{R(f)_{\mathfrak{o}}}\right)
$$

is satisfied for each drifter $\mathfrak{d}$ in an instable TFPL $f$ of excess at most 2 . Depending on which of the two inequalities $\mathfrak{d}$ satisfies it is moved to the left or to the right boundary where it then is deleted: let $f$ be a TFPL with boundary $(u, v ; w)$ where $\operatorname{exc}(u, v ; w) \leq 2$. A triple $(S(f), g(f), T(f))$ consisting of a semistandard Young tableau $S(f)$ of skew shape $\lambda\left(u^{+}\right) / \lambda(u)$, a stable TFPL $g(f)$ with boundary $\left(u^{+}, v^{+} ; w\right)$ and a semi-standard Young tableau $T(f)$ of skew shape $\lambda\left(v^{+}\right) / \lambda(v)$ is associated with $f$ as follows:

- If $f$ is stable, then set $g(f)=f, S(f)$ the empty semi-standard Young tableau of skew shape $\lambda(u) / \lambda(u)$ and $T(f)$ the empty semi-standard Young tableau of skew shape $\lambda(v)^{\prime} / \lambda(v)^{\prime}$.

- If in $f$ for each drifter $\mathfrak{d}$ it holds $R(f)_{\mathfrak{d}} \leq R_{1}\left(u^{R(f)_{\mathfrak{o}}}\right)$, then $g(f)=\operatorname{Right}(f)=\mathrm{WR}^{R(f)+1}(f)$, where the boundary of $\operatorname{Right}(f)$ is $\left(u^{+}, v ; w\right)$ for a $u^{+}$such that $\lambda(u) \subset \lambda\left(u^{+}\right), S(f)$ is the semi-standard Young tableau of skew shape $\lambda\left(u^{+}\right) / \lambda(u)$ corresponding to the sequence

$$
\lambda(u)=\lambda\left(u^{0}\right) \subseteq \lambda\left(u^{1}\right) \subseteq \cdots \subseteq \lambda\left(u^{R(f)}\right) \subseteq \lambda\left(u^{R(f)+1}\right)=\lambda\left(u^{+}\right),
$$

where $u^{r}$ denotes the left boundary word of $\mathrm{WR}^{r}(f)$ for each $0 \leq r \leq R(f)+1$, and $T(f)$ is the empty semi-standard Young tableau of skew shape $\lambda(v)^{\prime} / \lambda(v)^{\prime}$.

- If in $f$ for each drifter $\mathfrak{d}$ it holds $L(f)_{\mathfrak{d}} \leq L_{0}\left(v^{L(f)_{\mathfrak{o}}}\right)$, then set $g(f)=\operatorname{Left}(f)=\mathrm{WL}^{L(f)+1}(f)$, where the boundary of $\operatorname{Left}(f)$ is $\left(u, v^{+} ; w\right)$ for a $v^{+}$such that $\lambda(v) \subset \lambda\left(v^{+}\right), S(f)$ is the empty semi-standard Young tableau of skew shape $\lambda(u) / \lambda(u)$ and $T(f)$ is the semi-standard Young tableau of skew shape $\lambda\left(v^{+}\right)^{\prime} / \lambda(v)^{\prime}$ corresponding to the sequence

$$
\lambda(v)^{\prime}=\lambda\left(v^{0}\right)^{\prime} \subseteq \lambda\left(v^{1}\right)^{\prime} \subseteq \cdots \subseteq \lambda\left(v^{L(f)}\right)^{\prime} \subseteq \lambda\left(v^{L(f)+1}\right)^{\prime}=\lambda\left(v^{+}\right)^{\prime},
$$

where $v^{\ell}$ denotes the right boundary word of $\mathrm{WL}^{\ell}(f)$ for each $0 \leq \ell \leq L(f)+1$.

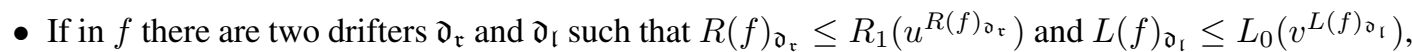
then $g(f)$ is the TFPL with boundary $\left(u^{+}, v^{+} ; w\right)$ for a $u^{+}$such that $\lambda(u) \subset \lambda\left(u^{+}\right)$and a $v^{+}$such that $\lambda(v) \subset \lambda\left(v^{+}\right)$obtained from $f$ as follows: the drifter $\mathfrak{d}_{\mathfrak{r}}$ is moved to the left boundary using the moves $M_{1}^{-1}, M_{2}^{-1}, M_{3}^{-1}$ and there replaced by a horizontal edge and the drifter $\mathfrak{d}_{\mathfrak{l}}$ is moved to the right boundary using the moves $M_{1}, M_{2}, M_{3}$ and there replaced by a horizontal edge. Furthermore, $S(f) R(f)_{\mathfrak{d}_{\mathfrak{l}}}$ is the semi-standard Young tableau of skew shape $\lambda\left(u^{+}\right) / \lambda(u)$ with entry $R(f)_{\mathfrak{d}_{\mathfrak{r}}}+1$ and $T(f)$ is the semi-standard Young tableau of skew shape $\lambda\left(v^{+}\right)^{\prime} / \lambda(v)^{\prime}$ with entry $L(f)_{\mathfrak{d}_{\mathfrak{l}}}+1$.

In Figure 4, the TFPL of excess 2 of Figure 7 and the triple $(S(f), g(f), T(f))$ associated with it are depicted. In the following, denote by $G_{\lambda, \lambda^{+}}$the set of semi-standard Young tableaux of skew shape $\lambda^{+} / \lambda$ with entries in the $i$-th column, if counted from right, restricted to $1,2, \ldots, i$ for Young diagrams $\lambda \subseteq \lambda^{+}$ which may have empty columns or rows. 


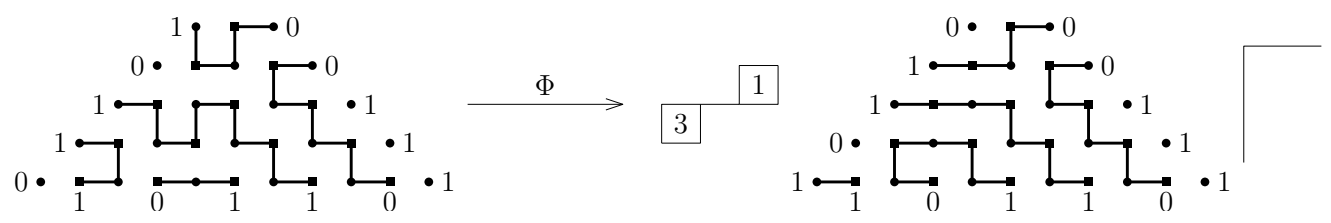

Fig. 8: An instable TFPL with boundary $(01101,00111 ; 10110)$ and the triple $(S(f), g(f), T(f))$ it is associated with.

Theorem 2 Let $u, v, w$ be words of the same length and with the same number of occurrences of one such that exc $(u, v ; w) \leq 2$. Then the map

$$
\begin{aligned}
\Phi: T_{u, v}^{w} & \longrightarrow \bigcup_{\substack{u^{+}: \lambda(u) \subseteq \lambda\left(u^{+}\right) \\
v^{+}: \lambda(v) \subseteq \lambda\left(v^{+}\right)}} G_{\lambda(u), \lambda\left(u^{+}\right)} \times S_{u^{+}, v^{+}}^{w} \times G_{\lambda(v)^{\prime}, \lambda\left(v^{+}\right)^{\prime}} \\
f & \longmapsto(S(f), g(f), T(f))
\end{aligned}
$$

is a bijection.

Corollary 4.1 The assertion of Theorem 1 immediately follows from Theorem 2

Proposition 4.2 Let $f$ be an instable TFPL of excess 2 that possesses two drifters $\mathfrak{d}$ and $\mathfrak{d}^{*}$. If $\mathfrak{d}$ is not incident to a vertex in $\mathcal{R}^{N}$ and no move $M_{1}, M_{2}$ or $M_{3}$ can be applied to $\mathfrak{d}$, then

$$
L(f)_{\mathfrak{o}}-L(f)_{\mathfrak{o}^{*}}=L_{0}\left(v^{L(f)_{\mathfrak{o}}}\right)-L_{0}\left(v^{L(f)_{\mathfrak{o}}}\right)+1 .
$$

An immediate consequence of Proposition 4.2 is that if in $f$ there are two drifters $\mathfrak{d}_{\mathfrak{r}}$ and $\mathfrak{d}_{\mathfrak{l}}$ such that $R(f)_{\mathfrak{d}_{\mathfrak{r}}} \leq R_{1}\left(u^{\left.R(f)_{\mathfrak{o}_{\mathfrak{r}}}\right)}\right.$ and $L(f)_{\mathfrak{d}_{\mathfrak{r}}} \leq L_{0}\left(v^{\left.L(f)_{\mathfrak{o}_{\mathfrak{r}}}\right)}\right.$, then $\mathfrak{d}_{\mathfrak{r}}$ can be moved to the left boundary using the moves $M_{1}^{-1}, M_{2}^{-1}, M_{3}^{-1}$ and $\mathfrak{d}_{\mathfrak{l}}$ can be moved to the right boundary using the moves $M_{1}, M_{2}, M_{3}$. The proof of Proposition 4.2 is done in terms of blue-red path tangles.

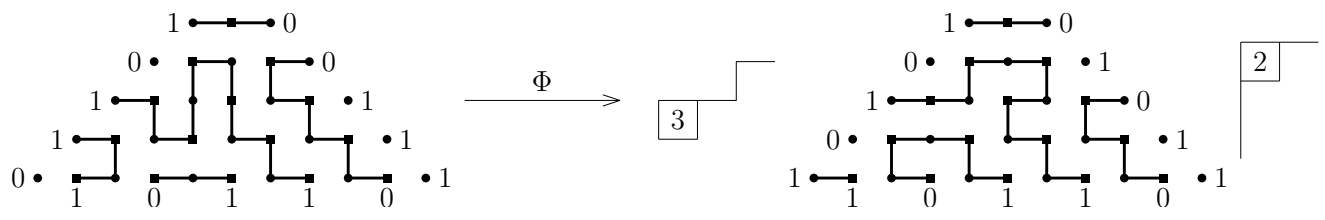

Fig. 9: An instable TFPL of excess 2 and the triple $(S(f), g(f), T(f))$ it is associated with by $\Phi$.

Proof of Theorem 2: Let $u, v, w$ be words of length $N$ with the same number of occurrences of one such that $\operatorname{exc}(u, v ; w) \leq 2$. Furthermore, let $f$ be an instable TFPL with boundary $(u, v ; w)$ and $(S(f), g(f), T(f))$ the image of $f$ under $\Phi$. Then $S(f) \in G_{\lambda(u), \lambda\left(u^{+}\right)}$for the following reason: let $c$ be a cell of the Young diagram of skew shape $\lambda\left(u^{+}\right) / \lambda(u)$ then its entry has to be $R(f)_{\mathfrak{d}}+1$ for a drifter $\mathfrak{d}$ in $f$. Furthermore, $R(f)_{\mathfrak{d}} \leq R_{1}\left(u^{R(f)_{\mathfrak{o}}}\right)$. Since $R_{1}\left(u^{R(f)_{\mathfrak{o}}}\right)$ is the number of columns to the right of $c$ in the skew shape $\lambda\left(u^{+}\right) / \lambda(u)$ the assertion follows. By analogous arguments, $T \in G_{\lambda(v)^{\prime}, \lambda\left(v^{+}\right)^{\prime}}$. 
Thus, it remains to prove that $\Phi$ is a bijection. This is done by giving the inverse map $\Psi$ : let $u^{+}$be a word such that $\lambda(u) \subseteq \lambda\left(u^{+}\right), v^{+}$be a word such that $\lambda(v) \subseteq \lambda\left(v^{+}\right), S \in G_{\lambda(u), \lambda\left(u^{+}\right)}, g \in S_{u^{+}, v^{+}}^{w}$ and $T \in G_{\lambda(v)^{\prime}, \lambda\left(v^{+}\right)^{\prime}}$. Furthermore, consider the sequences

$$
\lambda(u)=\lambda\left(u^{0}\right) \subseteq \lambda\left(u^{1}\right) \subseteq \cdots \subseteq \lambda\left(u^{R}\right) \subseteq \lambda\left(u^{R+1}\right)=\lambda\left(u^{+}\right)
$$

corresponding to $S$, where $R+1$ is the largest entry of $S$, and

$$
\lambda(v)^{\prime}=\lambda\left(v^{0}\right)^{\prime} \subseteq \lambda\left(v^{1}\right)^{\prime} \subseteq \cdots \subseteq \lambda\left(v^{L}\right)^{\prime} \subseteq \lambda\left(v^{L+1}\right)^{\prime}=\lambda\left(v^{+}\right)^{\prime}
$$

corresponding to $T$, where $L+1$ is the largest entry of $T$. Then associate $(S, g, T)$ with a TFPL $\Psi(S, g, T)$ with boundary $(u, v ; w)$ as follows:

- If $u^{+}=u$ and $v^{+}=v$, then $\Psi(S, g, T)=g$.

- If $u^{+}>u$ and $v^{+}=v$, then set $\Psi(S, g, T)=\left(\mathrm{WL}_{u^{0}} \circ \mathrm{WL}_{u^{1}} \circ \cdots \circ \mathrm{WL}_{u^{R-1}} \circ \mathrm{WL}_{u^{R}}\right)(g)$.

- If $u^{+}=u$ and $v^{+}>v$, then set $\Psi(S, g, T)=\left(\mathrm{WR}_{v^{0}} \circ \mathrm{WR}_{v^{1}} \circ \cdots \circ \mathrm{WR}_{v^{L-1}} \circ \mathrm{WR}_{v^{L}}\right)(g)$.

- If $u^{+}>u$ and $v^{+}>v$, then $\Psi(S, g, G)$ is the TFPL obtained from $g$ as follows: a drifter $\mathfrak{d}_{\mathfrak{r}}$ incident to $L_{i_{j}+1}$ is inserted, whereas the horizontal edge incident to $L_{i_{j}}$ is deleted, if $j$ is the column counted from right that contains the one cell in the Young diagram of skew shape $\lambda\left(u^{+}\right) / \lambda(u)$ and $i_{j}$ is the index of the $j$-th one in $u^{+}$; a drifter $\mathfrak{d}_{\mathfrak{l}}$ incident to $R_{i_{j}^{\prime}-1}$ is inserted, whereas the horizontal edge incident to $R_{i_{j}^{\prime}}$ is deleted, if $j$ is the column counted from right that contains the one cell in the Young diagram of skew shape $\lambda\left(v^{+}\right)^{\prime} / \lambda(v)^{\prime}$ and $i_{j}^{\prime}$ is the index of the $j$-th zero in $v^{+}$. Then $\mathfrak{d}_{\mathfrak{r}}$ is moved $R$ times by a move $M_{1}, M_{2}$ or $M_{3}$ and $\mathfrak{d}_{\mathfrak{l}}$ is moved $L$ times by a move $M_{1}^{-1}, M_{2}^{-1}$ or $M_{3}^{-1}$.

It can easily be seen that $\Psi$ is the inverse map of $\Phi$.

\section{References}

[1] S. Beil, I. Fischer and P. Nadeau. Wieland drift for triangular fully packed loop configurations, Elect. J. Comb., 22(1), 2015

[2] F. Caselli, C. Krattenthaler, B. Lass and P. Nadeau. On the number of fully packed loop configurations with a fixed associated matching. Elect. J. Comb., 11(2), 2004.

[3] I. Fischer, P. Nadeau. Fully Packed Loops in a triangle: matchings, paths and puzzles. J. Combinanorial Theory Ser. A, 130:64-118, 2015.

[4] P. Nadeau. Fully Packed Loop Configurations in a Triangle and Littlewood-Richardson coefficients. J. Combinanorial Theory Ser. A, 120(8):2137-2147, 2013.

[5] J. Thapper. Refined counting of fully packed loop configurations. Séminaire Lotharingien de Combinatoire, 56:B56e:27, 2007.

[6] B. Wieland. A large dihedral symmetry of the set of alternating sign matrices. Elect. J. Comb., 7(1-3), 2000. 